\title{
Aspectos Epidemiológicos da Leishmaniose Visceral
}

\author{
Epidemiological Aspects of Visceral Leishmaniasis
}

Aspectos Epidemiológicos de la Leishmaniasis Visceral

\begin{abstract}
Eliana do Socorro Monteiro dos Santos ${ }^{1}$, Adilson Mendes de Figueiredo Júnior ${ }^{1 *}$, Gabriela Barbosa Maia dos Santos ${ }^{1}$, Raimunda de Freitas da Silva ${ }^{1}$, Elizangela Fonseca de Mendonça ${ }^{1}$, Rubia Rodrigues Neves ${ }^{1}$, Marcio Nonato da Costa Borges ${ }^{2}$.
\end{abstract}

\section{RESUMO}

Objetivo: O objetivo deste trabalho foi descrever, através do levantamento da vigilância entomológica, a presença de Lutzomyia Longipalpis e Lutzomyia Cruzi, e as atribuições da Enfermagem frente aos casos da LV. Metodologia: Trata-se de uma pesquisa epidemiológica de caráter quantitativo descritivo e exploratório, onde as informações utilizadas nesta pesquisa foram colhidas por meio de dados dos relatórios de casos da LV obtidos da Prefeitura municipal de Belém e utilizou-se para discussão dos dados a Biblioteca Virtual em Saúde (BVS) onde selecionou-se a base de dados da Literatura Latino-Americana e do Caribe em Ciências da Saúde (LILACS). Para realizar a coleta dos artigos científicos utilizamos os descritores Enfermagem, Leishmaniose e Leishmaniose Visceral. Resultados: Ficou evidente que o perfil da LV é caracterizado em adultos do sexo masculino que fica localizado na zona urbana, e a espécie com maior quantidade encontrada foi a Lutzomyia Longipalpis. Conclusão: O enfermeiro frente a LV é indispensável para que haja prevenção, promoção e reabilitação da saúde do indivíduo.

Palavras-Chave: Enfermagem, Leishmaniose Visceral, Leishmaniose.

\begin{abstract}
Objective: The purpose of this study was to describe the presence of Lutzomyia Longipalpis and Lutzomyia Cruzi, and the attributions of Nursing to the cases of VL. Methodology: This is an epidemiological research of descriptive and exploratory quantitative character, where the information used in this research was collected through data from the LV case reports obtained from the Municipality of Belém, and the Library was used to discuss the data (VHL) where the Latin American and Caribbean Literature in Health Sciences database (LILACS) was selected. To perform the collection of scientific articles we use the descriptors Nursing, Leishmaniasis and Visceral Leishmaniasis. Results: It was evident that the LV profile is characterized in male adults that is located in the urban zone, and the species with the highest amount found was Lutzomyia Longipalpis. Conclusion: The nurse facing VL is indispensable for the prevention, promotion and rehabilitation of the individual's health.
\end{abstract}

Keywords: Nursing, Visceral Leishmaniasis, Leishmaniasis.

\section{RESUMEN}

Objetivo: El objetivo de este trabajo fue describir, a través del levantamiento de la vigilancia entomológica, la presencia de Lutzomyia Longipalpis y Lutzomyia Cruzi, y las atribuciones de la Enfermería frente a los casos de la LV. Metodología: Se trata de una investigación epidemiológica de carácter cuantitativo descriptivo y exploratorio, donde las informaciones utilizadas en esta investigación fueron recolectadas por medio de datos de los informes de casos de la LV obtenidos del Ayuntamiento municipal de Belém y se utilizó para discusión de los datos la Biblioteca Virtual en Salud (BVS) donde se seleccionó la base de datos de la Literatura Latinoamericana y del Caribe en Ciencias de la Salud (LILACS). Para realizar la recolección de los artículos

\footnotetext{
${ }^{1}$ Escola Superior da Amazônia (ESAMAZ). Belém-Pará. *E-mail: adilsonmdfj@hotmail.com

²Universidade Federal do Pará (UFPA). Belém-Pará.
}

SUBMETIDO EM: 11/2018 
científicos utilizamos los descriptores Enfermería, Leishmaniosis y Leishmaniosis Visceral. Resultados: Es evidente que el perfil de la LV se caracteriza en adultos del sexo masculino que se encuentra en la zona urbana, y la especie con mayor cantidad encontrada fue la Lutzomyia Longipalpis. Conclusión: El enfermero frente a LV es indispensable para que haya prevención, promoción y rehabilitación de la salud del individuo.

Palabras-clave: Enfermería, Leishmaniosis Visceral, La leishmaniasis.

\section{INTRODUÇÃO}

A Leishmaniose Visceral, popularmente conhecida como calazar, é uma doença grave, infecciosa e sistêmica causada por protozoário do tipo Leishmania chagasi, e é transmitida pelo vetor Lutzomyia Cruzi. A Leishmaniose Visceral apresenta como seu principal reservatório no meio ambiente urbano o cão doméstico, onde esta doença é evidenciada em todos os estados do Brasil, assim como em outros países da América Latina (BRASIL, 2006).

A cada ano são registrados quase dois milhões de novos casos dessa zoonose no mundo, e segundo estimativas da organização mundial da saúde (OMS), cerca de $90 \%$ dos casos na América Latina ocorrem no Brasil, onde, em uma média anual, três mil casos da doença são evidenciados (ORTIZ RC, ANVERSA L, 2015).

A doença deixa de ter um caráter de transmissão residual e passa a se expandir em dimensões maiores pelo país, tais dimensões passam a ser verificadas a partir dos anos de 1980, com o registro de transmissão em áreas urbanizadas de grandes cidades vê-se a disseminação da doença que partiu da região nordeste, se proliferando na região norte, e depois na região centro-oeste e por fim o sudeste, causando epidemias urbanas e consequentemente a incidência de indivíduos contaminados e aumento no índice de letalidade da doença. Algumas pessoas não apresentam sintomas, mas em outras, os sintomas podem incluir febre, perda de peso e inchaço do baço ou fígado (hepatoesplenomegalia). Existem medicamentos para eliminar os parasitas, mas se não forem tratados, os casos graves costumam ser fatais (BRASIL, 2017).

No Brasil a Leishmaniose Visceral é zooantroponose peri-urbana e rural, no âmbito das residências o cão é um importante reservatório do agente para a proliferação e contaminação dos indivíduos. No ambiente silvestre a raposa e outros animais canídeos são os reservatórios que agem como hospedeiros naturais. $O$ vetor da doença é o Lutzomyia Longipalpis cuja distribuição se supõe em áreas endêmicas, onde a sua proliferação age em locais húmidos e de baixa luminosidade, onde, em locais de considerável arborização e intensidade pluviométrica se faz importante para a vivência deste inseto (ORTIZ RC, ANVERSA L, 2015). O objetivo deste trabalho foi descrever, através do levantamento da vigilância entomológica, a presença de Lutzomyia Longipalpis e Lutzomyia Cruzi, e as atribuições da Enfermagem frente aos casos da LV.

\section{MÉTODOS}

Este estudo trata-se de uma pesquisa epidemiológica de caráter quantitativo descritivo e exploratório baseado em dados da leishmaniose visceral no município de Belém na localidade de Cotijuba.

A pesquisa quantitativa se debruça em estudar de forma aproximada o alvo ou objeto de estudo em caráter estatístico sob a orientação de dados numéricos, indicadores, fórmulas matemáticas ou estatísticas. Como exemplo de estudos quantitativos, o autor cita o estudo de análise de relatório de campo, o estudo de caso tabulado com informações numéricas, dentre outros; como dito antes, não há uma fórmula puramente numérica, onde a pesquisa quantitativa é bastante influenciada pelo próprio pesquisador. Se destaca na pesquisa quantitativa a análise que se faz sistematicamente das semelhanças e diferenças que agem interrelacionadas no conjunto de todas as informações levantadas na pesquisa (GIL, 2008).

Os critérios de inclusão utilizados na pesquisa foram artigos nacionais e completos, que contemplem a temática do estudo e escrito no idioma português. Os critérios de exclusão utilizados na pesquisa foram 
artigos que não contemplavam a temática do estudo, artigos incompletos, artigos duplicados e que estão em outro idioma. O levantamento de dados para a RIL (Revisão Integrativa da Literatura) foi realizado através do acesso na Biblioteca Virtual da Saúde, usando os descritores em saúde definidos.

\section{RESULTADO E DISCUSSÃO}

A doença tem uma evolução crônica, grave e de alta letalidade, visto que a falta do tratamento adequado e precoce poderá submeter para a evolução desta doença chegando ao óbito do paciente. Seu aspecto clínico e de caráter epidemiológico, assim como a investigação de como se dá a evolução desta endemia se apresenta sob as mais diversas complexidades naturais, geográficas e culturais em cada região é submetida, porém, no que tange a sua forma de transmissão as características são basicamente iguais, ocorrem pela picada do mosquito vetor Lutzomyia Longipalpis e o Lutzomyia Cruzi denominado de Flebotomíneos, que é o principal transmissor da doença em questão (CONITEC, 2016).

Foi possível caracterizar a análise de uma primeira notificação desta doença que fora descoberta na ilha de Cotijuba, localidade esta que pertence ao município de Belém, o que proporcionará uma análise do conhecimento epidemiológico no trato deste agravo, uma vez que a disseminação da doença poderá atingir um quantitativo maior de indivíduos, o que poderá ter alcances de grande magnitude geográfica. Por isso, a identificação e o controle epidemiológico se faz importante neste processo, uma vez que o trabalho de promoção da saúde deverá ser eficiente no que tange a saúde geral da sociedade.

Mediante a esse contexto destaca-se que os indivíduos do gênero masculino estão mais propícios a LV em decorrência da falta de atenção em relação aos fatores de riscos, os meios preventivos e não procura de uma unidade de saúde, todavia as mulheres procuram mais as unidades de saúde do que os homens. De acordo os autores a zona urbana estão ligados a higiene ambiental da população (PIRES et al, 2016).

Ficou evidente que cerca de $90 \%$ da população que desenvolve a LV, que não procura ajuda médica evoluiu a óbito, essa doença é transmitida na zona urbana principalmente quando fêmeas contaminadas picam os cães e logo após picam o ser humano. Em nosso país é mais comumente encontrar a espécie Lutzomyia Longipalpis. Em virtude disso o melhor meio para prevenir é fazer uso das medidas como realizar limpeza periódica de terrenos, desprezar o lixo em local adequado, uso de inseticidas e outros (CONITEC, 2016).

Foram encontrados 426 espécimes, os quais são pertencentes a 7 espécies de flebotomíneos. A espécie mais encontrada foi a Lutzomyia Longipalpis correspondente a $52.8 \%$, seguida das espécies Lutzomyia Oswaldoi correspondente a 13,4\% Lutzomyia Flaviscutellata correspondente a 5,4\%, Lutzomyia Nordestina correspondente a 10,8\%, Lutzomyia Yuilli Yuilli correspondente a 8,7\%, Brumptomya Cunhai correspondente a 2,1\%, e a Psychodopygus davisi correspondente a 6,8\% (Tabela 1).

Tabela 1 - Presença de Lutzomyia Longipalpis na ilha de Cotijuba em Belém-PÁ em 2016.

\begin{tabular}{l|l|l|l}
\hline \hline Espécie & Macho & Fêmea & Total \\
\hline \hline Lutzomyia Longipalpis & 169 & 56 & 225 \\
Lutzomyia Oswaldoi & 42 & 15 & 57 \\
Lutzomyia Flaviscutellata & 16 & 7 & 23 \\
Lutzomyia Nordestina & 33 & 13 & 46 \\
Lutzomyia Yuilli Yuilli & 25 & 12 & 37 \\
Brumptomya Cunhai & 5 & 4 & 9 \\
Psychodopygus davisi & 17 & 12 & 29 \\
\hline Total & 307 & 119 & 426 \\
\hline
\end{tabular}

Fonte: Laboratório de Entomologia/SESMA, 2016. 
Os programas de prevenção vêm com intuito de minimizar este problema de Saúde Pública, os órgãos governamentais nos níveis Federal, Estadual e Municipal vêm propondo alternativas para melhorar as condições de saúde da população por meio do desenvolvimento de assistência à saúde com qualidade e resolubilidade. Neste sentido o MS pela Portaria № 5 de 21 de fevereiro de 2006, incluiu a Leishmaniose Visceral como uma das doenças de notificação compulsória, promovendo assim o aprimoramento do Programa de Vigilância e Controle da Leishmaniose Visceral (PVCLV), com objetivo de desenvolver e aplicar medidas educativas e preventivas (BRASIL, 2006).

Dentre as medidas preventivas deste programa, estabeleceram-se prioridades e estratégias de controle, visando a detectar e tratar os casos sintomáticos e assintomáticos em humanos, além da detecção e eliminação (eutanásia) de cães infectados, o que ocorre após a confirmação por exame parasitológico e/ou sorológico, saneamento ambiental, redução e controle dos flebotomíneos, além da estratégia de educação em saúde da população (CONITEC, 2016).

O papel do profissional de enfermagem consiste em programar medidas educativas, com a finalidade de minimizar os números de casos causados pela LV (Quadro 2). Contudo pode ser destacado que os profissionais da área da saúde em especial os enfermeiros são fundamentais para tentar sensibilizar a população quanto os fatores de riscos e os meios diagnósticos (ORTIZ RC, ANVERSA L, 2015).

Todavia o enfermeiro atua mediante as formas de prevenção fazendo uso da educação em saúde seja em uma comunidade, ou até mesmo nas escolas por meio de palestras, debates para promover maior conhecimento. Porém os enfermeiros promovem atividades e identifica as principais sintomatologias da LV auxiliando a população quanto às formas de transmissão e tratamento (CAVALCANTE IJM, VALE MR, 2014).

Para auxiliar os pacientes diagnosticados com a LV os enfermeiros fazem uso dos diagnósticos de enfermagem, objetivando uma assistência eficaz e segura aos indivíduos. O papel da enfermagem consiste em esclarecer as possíveis reações das medicações visto que em alguns pacientes surgem reações (AGUIAR PF, RODRIGUES RK, 2017).

De acordo com os estudos realizados ficou evidente que em muitos casos a LV não surge sinais e sintomas, entretanto em outros casos são notórios, a LV se não tratada poderá levar a as pessoas doentes a óbito mediante a esse contexto os enfermeiros identificam os sintais e sintomas, realiza medidas de prevenção, auxilia no tratamento, monitora e avalia as ações de saúde (BARBOSA I, 2016).

Quadro 2 - Caracterização do Papel do enfermeiro frente a LV.

\begin{tabular}{|c|l|}
\hline CÓDIGO & PAPEL DO ENFERMEIRO FRENTE A LV \\
\hline 01 & Metodologias de educação e Prevenção \\
\hline 02 & Atividades para o controle \\
\hline 03 & Educação em saúde \\
\hline 04 & $\begin{array}{l}\text { Auxiliar no planejamento, monitoramento e avaliação das ações em saúde, } \\
\text { direcionando as intervenções para diminuir as iniquidades }\end{array}$ \\
\hline 05 & Sinais clínicos e medidas preventivas \\
\hline 06 & Ações de controle \\
\hline 07 & O enfermeiro atua frente às estratégias de prevenção \\
\hline 08 & Elaboração dos diagnósticos de enfermagem \\
\hline
\end{tabular}

Fonte: Dados da pesquisa, 2018.

No tocante a disseminação de doenças transmissíveis por vetores que se encontram tanto em localidades de características rurais e urbanas nas cidades que formam a nação brasileira, fica evidente o trato quanto a informação e conhecimento da sociedade no que tange aos devidos cuidados com a proliferação, 
contaminação e possível adoecimento dos indivíduos. Disto isto, uma variedade de agravos que são transmitidos por vetores como insetos, cães, moluscos, roedores, dentre outros. E que, portanto, destaca-se o conhecimento da população para o "enfrentamento" de tais doenças, o que demonstra a importância conjunta do setor público juntamente com a sociedade.

\section{CONCLUSÃO}

Por intermédio desse estudo foi possível identificar os aspectos epidemiológicos da Leishmaniose Visceral, em virtude disso o profissional enfermeiro atua frente à prevenção por meio de palestras voltadas para a população, com intuito de sensibilizar os indivíduos quanto aos meios preventivos e aos fatores de riscos para que possa garantir maior promoção à saúde e melhor qualidade de vida. É importante destacar que as estratégias empregadas pelo enfermeiro certamente diminuirá o número de casos dessa patologia, visto que a espécie mais encontrada na ilha de Cotijuba foi a Lutzomyia Longipalpis, todavia essa é uma doença que pode levar a óbito quando não tratada independente da faixa etária dos indivíduos, uma vez que os estudos comprovam que o gênero masculino está mais favorável. Contudo, as pessoas que residem na zona urbana têm maiores chances de ser contaminado pela LV, por isso é necessário que os indivíduos que estão em locais de riscos ou que apresentam algum sintoma procurem ajuda, desta forma, é relevante destacar os profissionais de enfermagem os quais são essenciais neste contexto para programar os cuidados aos que necessitam.

\section{REFERÊNCIAS}

1. AGUIAR PF, RODRIGUES RK. Leishmaniose visceral no Brasil: artigo de revisão. Montes Claros, v. 19 , n.1 - jan./jun. 2017.

2. BARBOSA I. Leishmaniose visceral humana no município de natal: análise clínico-epidemiológica e espacial. Revista Ciência Plural, v. 2, n. 1, p. 89-101, 30 ago. 2016.

3. BLANCO VR, NASCIMENTO-JÚNIOR NM. Leishmaniose: Aspectos Gerais Relacionados com a Doença, o Ciclo do Parasita, Fármacos Disponíveis, Novos Protótipos e Vacinas. Rev. Virtual Quim., 2017, 9 (3), 861-876. Data de publicação na Web: 24 de abril de 2017

4. BRASIL. Conselho Federal de Enfermagem. Dispõe sobre a Sistematização da Assistência de Enfermagem - SAE - nas Instituições de Saúde Brasileiras. Resolução COFEN-272/2002 - Revogada pela Resolução COFEN no $358 / 2009$.

5. BRASIL. Ministério da Saúde. A presente Resolução incorpora, sob a ótica do indivíduo e das coletividades, referenciais da bioética, tais como, autonomia, não maleficência, ineficiência, justiça e equidade, dentre outros, e visa a assegurar os direitos e deveres que dizem respeito aos participantes da pesquisa, à comunidade científica e ao Estado. Resolução no 466 , de 12 de dezembro de 2012. Brasília: Conselho Nacional de Saúde.

6. BRASIL. Ministério da Saúde. Inclui doenças na relação nacional de notificação compulsória, define doenças de notificação imediata, relação dos resultados laboratoriais que devem ser notificados pelos Laboratórios de Referência Nacional ou Regional e normas para notificação de casos. Portaria ํㅡ 5, de 21 de fevereiro de 2006. Brasília: Legislação Federal.

7. BRASIL. Ministério da Saúde. Manual de Vigilância e Controle da Leishmaniose Visceral, 2014.

8. BRASIL. Portal do Governo Federal. Casos de leishmaniose caem no País, mas doença ainda requer atenção, 2017.

9. CAVALCANTE ÍJM, VALE MR. Aspectos epidemiológicos da leishmaniose visceral (calazar) no Ceará no período de 2007 a 2011. Rev. bras. epidemiol., São Paulo, v. 17, n. 4, p. 911-924, dez. 2014.

10. COFEN. Conselho Federal de Enfermagem. Dispõe sobre a Sistematização da Assistência de Enfermagem e a implementação do Processo de Enfermagem em ambientes, públicos ou privados, em que ocorre o cuidado profissional de Enfermagem, e dá outras providências. Resolução COFEN-358/2009.

11. CONITEC. Comissão Nacional de incorporação de Tecnologia no SUS. Proposta de elaboração protocolo Clínico e diretrizes terapêuticas: Escopo Leishmaniose Visceral. Julho/2016.

12. COSTA KFL. Percepção e diagnóstico da leishmaniose visceral canina em áreas ribeirinhas na cidade de Mossoró, Rio Grande do Norte. 2003. 93 f. Dissertação. (Mestrado em Ambiente, Tecnologia e Sociedade) - Programa de pós-graduação em ambiente, tecnologia e sociedade da UFERSA. Mossoró, 2016.

13. GIL AC. Métodos e técnicas de pesquisa social. São Paulo: Atlas, 2008.

14. GONTIJO CMF, MELO MN. Leishmaniose Visceral no Brasil: quadro atual, desafios e perspectivas. Revista Brasileira de Epidemiologia. Vol. 7, № 3, 2004.

15. ORTIZ RC, ANVERSA L. Epidemiologia da leishmaniose visceral em Bauru, São Paulo, no período de 2004 a $2012:$ um estudo descritivo. Epidemiol. Serv. Saúde 24 (1) Jan-Mar 2015.

16. PIRES BS. et al. Fatores epidemiológicos da Leishmaniose visceral humana no Brasil, 2008-2015. Simpósio de TCC e Seminário de IC, 2016. 